\title{
Remote Ultrasonic Imaging of a Wire Arc Additive Manufactured Ti-6Al-4V Component using Laser Induced Phased Array
}

\author{
Peter Lukacs \\ Department of Electronic and Electrical \\ Engineering \\ University of Strathclyde \\ Glasgow, UK \\ peter.lukacs@strath.ac.uk \\ Stewart Williams \\ Welding Engineering And Laser \\ Processing \\ University of Cranfield \\ Cranfield, UK \\ s.williams@cranfield.ac.uk
}

\author{
Geo Davis \\ Department of Electronic and Electrical \\ Engineering \\ University of Strathclyde \\ Glasgow, UK \\ geo.davis@strath.ac.uk \\ Charles N. MacLeod \\ Department of Electronic and Electrical \\ Engineering \\ University of Strathclyde \\ Glasgow, UK \\ charles.macleod@strath.ac.uk
}

\author{
Theodosia Stratoudaki \\ Department of Electronic and Electrical \\ Engineering \\ University of Strathclyde \\ Glasgow, UK \\ t.stratoudaki@strath.ac.uk \\ Anthony Gachagan \\ Department of Electronic and Electrical \\ Engineering \\ University of Strathclyde \\ Glasgow, UK \\ a.gachagan@strath.ac.uk
}

\begin{abstract}
Additive manufacturing (AM) has been revolutionizing the manufacturing industry due to its ability to significantly reduce waste and produce components with intricate shapes. Laser Ultrasonics (LU) is a non-contact and couplant free method to generate and detect ultrasound. LU can accommodate complex component shapes; thus, it has the potential to provide a reliable in-process inspection method for AM components. In recent years the development of Laser Induced Phased Arrays (LIPAs) helped overcome the inherently low signal amplitudes of $\mathrm{LU}$ at the non-destructive, thermoelastic regime. In this paper, the Full Matrix Capture data acquisition method is used and a LIPA of 68 elements is synthesized in post processing. The Total Focusing Method imaging algorithm is applied for ultrasonic imaging. The technique is demonstrated on a highly scattering titanium alloy Wire Arc Additive Manufactured (WAAM) component producing high quality ultrasonic images, accurately imaging defects at depths up to $10 \mathrm{~mm}$ below the inspection surface.
\end{abstract}

Keywords-laser ultrasonics, ultrasonic imaging, additive manufacturing, remote sensing

\section{INTRODUCTION}

In Additive Manufacturing (AM), layers upon layers of material are deposited in order to produce a component. AM can create objects with more complex geometries while being more cost-effective than subtractive manufacturing techniques [1]. The emergence of AM created the necessity for new Non-Destructive Testing (NDT) techniques for accurate inspection of AM components. In this field, significant advancements have been made including robotic inspection towards NDT automation and wheel probes capable of adapting to curved surfaces commonly encountered in AM [2]-[3]. While these advancements have been expanding inspection capabilities for NDT, transducer-based phased arrays require to be in contact with and coupled to the test object, complicating applications for in-process inspection, complex shapes and automation.

In Laser Ultrasonics (LU), ultrasonic waves are generated and detected using laser beams [4]. Lasers can be deployed remotely and used in places of restricted access, don't require

The work presented in this paper was supported by the UK Engineering and Physical Sciences Research Council [Grant reference EP/T012862/1, EP/R513349/1, EP/R027218/1] coupling to the test object and can adapt to complex shapes [5]. These features have made laser ultrasound attractive for NDT inspection of additive manufactured components [6]-[9]. Techniques such as Synthetic Aperture Focusing Technique [6] and B-scan imaging [7] have been successfully demonstrated on additively manufactured components; the former in ablative regime, causing surface damage, but achieving higher signal amplitudes, while the latter in the lower amplitude, truly non-destructive thermoelastic regime.

In recent years the development of Laser Induced Phased Arrays [10] (LIPAs) has greatly advanced the ultrasonic imaging capabilities of laser ultrasonics by adopting the Full Matrix Capture (FMC) and the Total Focusing Method (TFM). LIPAs are synthetic arrays, where generation and detection element combinations are achieved by scanning a single generation and a detection laser independently of each other. The focusing and steering action of the LIPA is done in post processing and a variety of imaging algorithms can be used. It is because of the imaging and steering of the synthetically composed LIPA that increased Signal-to-Noise Ratio (SNR) is achieved, addressing the limitations of conventional LU techniques. In a recent publication, LIPAs have demonstrated successful detection and characterisation of nested features in an Aluminium AM component made using Selective Laser Melting (SLM). The cylindrical features were as small as $0.2 \mathrm{~mm}$ in diameter and were located as deep as $26 \mathrm{~mm}$ below the inspection surface [9].

In this paper we present, laser ultrasonic imaging of a highly scattering Wire Arc Additive Manufactured (WAAM) component, made of Ti-6Al-4V titanium alloy. The novelty of the presented paper is that, for the first time, remote, nondestructive, ultrasonic inspection is attempted using a LIPA on a WAAM component and on a highly scattering material such as the titanium alloy.

\section{METHODS}

\section{A. Laser Ultrasound}

In LU, a small area of the test object is rapidly heated by a pulsed laser. The expansion caused by this heating creates stresses and strains producing acoustic waves [4]. All wave 
modes, bulk and surface waves are excited at the same time. The temporal characteristics of the generated ultrasonic waves are those of the laser pulse. Lasers with pulse duration at the nanosecond regime are typically used for generation of ultrasound, exciting waves with spectral content from DC up to hundreds of $\mathrm{MHz}$ [4]. In ultrasonic imaging, wideband signals allow for digital filtering in post-processing on the dataset, improving detectability at larger depths and resolution of the resultant images [10]. It is important to note that an ideal frequency range is application-specific, and it is a trade-off between low attenuation and scattering at lower frequencies and improved resolution at higher frequencies.

Laser induced ultrasound waves have different directivity and sensitivity patterns compared to those in transducers. The longitudinal and shear waves' directivity patterns in titanium, for the non-destructive, thermoelastic regime, are shown in Fig. 1. Fig. 1 is a composite polar graph showing only half branch of each wave mode, as the directivity patterns exhibit symmetry along the surface normal. As can be seen in this graph, there is minimal laser-generated ultrasound waves normal to the component surface. This applies to both longitudinal and shear wave excitation [11]. The amplitudes of the generated wave modes in Fig. 1 are indicated relative to each other. In the case of titanium, this would correspond to the longitudinal wave amplitude being $\sim 0.2$ times of the shear [12].

\section{B. Full Matrix Capture Data Acquisition}

The Full Matrix Capture is a data acquisition method used in ultrasonic phased arrays. The method captures an A-scan signal from every single generation and detection element combination of the array [13]. By doing so, a large volume of data is captured, which contains almost all the possible information that can be captured from a single position of the phased array. In contrast to some other scanning methods such as the focused B-scan or the sectoral scan, no beam steering or focusing is performed at the time of data acquisition. In order to produce ultrasonic images, the post-processing of the Full Matrix can be carried out by a wide range of imaging algorithms, from which the most commonly used is the delayand-sum Total Focusing Method (TFM) [13].

The TFM algorithm is considered a benchmark in NDT because of its ability to produce higher quality images compared to B-scan and the Synthetic Aperture Focusing Technique [14]. This has also been demonstrated for LIPAs in aluminum [10]. The TFM initially constructs a grid consisting of user-defined pixels. Using an FMC dataset, TFM

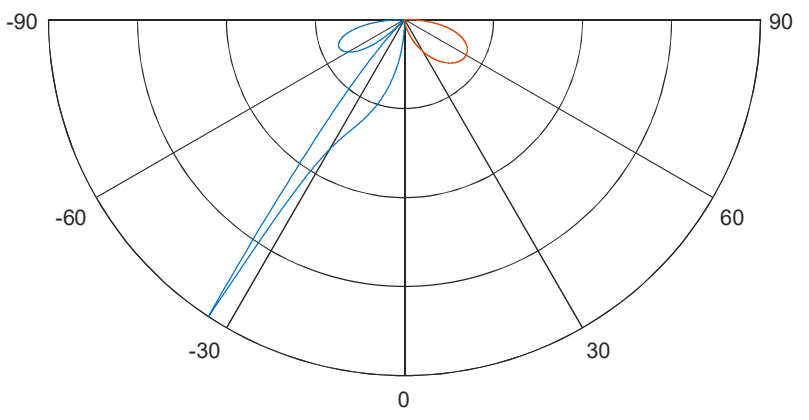

Fig. 1. Composite polar graph of laser generated directivity patterns, at the thermoelastic regime, in titanium: Left half-side of polar plot shows the directivity pattern of the shear wave and right half-side of polar plot shows the directivity pattern of the longitudinal wave. Relative amplitudes indicated, normalized to shear amplitude. synthetically focuses on each pixel of the grid in postprocessing. The pixel intensity of a TFM image can be calculated as defined by:

$$
I(x, z)=\left|\sum_{t x=1}^{n} \sum_{r x=1}^{n} S\left(\frac{d_{t x}+d_{r x}}{c}\right)\right|
$$

where $S$ is each A-scan signal of the Full Matrix dataset, $c$ is the acoustic velocity in the material, and each pixel is summed over every generation (tx) and detection (rx) points. Variables $d_{t x}$ and $d_{r x}$ are the distances between the pixel-transmitter, and pixel-receiver, respectively and are defined by (2) and (3).

$$
\begin{aligned}
& d_{t x}=\sqrt{\left(x_{t x}-x\right)^{2}+z^{2}} \\
& d_{r x}=\sqrt{\left(x_{r x}-x\right)^{2}+z^{2}}
\end{aligned}
$$

Equation (1) is the TFM expression developed for image processing of data captured using transducer-based phased arrays that have omni-directional directivity. In the case of laser ultrasonics, (3) had to be adapted to reflect the directivity patterns at the thermoelastic regime, as shown in Fig. 1. This is done by introducing apodization terms where the contribution of each waveform is weighted by the transmit and receive directivity functions at each image point. [15]. In this case, (1) becomes:

$$
I(x, z)=\left|\sum_{t x=1}^{n} \sum_{r x=1}^{n} Z_{t x}(x, z) * Z_{r x}(x, z) S\left(\frac{d_{t x}+d_{r x}}{c}\right)\right|
$$

Where $Z_{t x}$ and $Z_{r x}$ are apodization terms as defined in [15], based on the directivity and sensitivity patterns (Fig. 1).

The produced image was further enhanced by normalizing the TFM image with the sensitivity image. The latter describes the amplitude expected from a perfect point target (i.e. scattering matrix equal to unity) as a function of position. The normalized image has uniform sensitivity but non-uniform noise, as opposed to the initial TFM image which has uniform noise but non-uniform sensitivity [10]. The normalization process can be described by the following equation:

$$
N(x, z)=\frac{I(x, z)}{E(x, z)}
$$

Where $I(x, z)$ is the TFM image defined by (3) and $E(x, z)$ is the sensitivity image of the system at each individual pixel on the TFM grid. This sensitivity image is calculated by assuming perfect uniform scatterer at each pixel as described in [10].

As focusing is carried out on every pixel, TFM images have much greater signal-to-noise ratio and resolution when compared to some previous imaging techniques such as the Bscan. However, data storage size and processing times are also significantly increased [13].

\section{Synthetic Laser Ultrasonic Arrays}

A conventional, transducer-based array with various elements, each of them capable of generating and detecting ultrasound can easily and rapidly utilize FMC as a data acquisition method, where each transducer element is fired in turn and parallel detection is done from all elements. The same ideology would be difficult to replicate for laser ultrasonics in a practical setting due to the requirement of several laser generation/detection systems, leading to hardware constraints.

In order to overcome this challenge, LIPAs are implemented as synthetic arrays where the focusing and steering of ultrasound are done in post processing. A LIPA 
uses the superposition theorem to synthesize a phased array by scanning one generation and one detection laser independently of each other. Since the Full Matrix is captured, the advantage is that a range of imaging algorithms can be applied to the same data set. The disadvantage is the long data acquisition times due to the mechanical scanning of the lasers as well as averaging needed to compensate for the low amplitudes of laser ultrasonics.

\section{EXPERIMENTAL SETUP AND SAMPLE}

\section{A. Experimental Setup}

The experimental setup is shown in Fig. 2. Laser ultrasound generation is performed using a Q-switched $\mathrm{Nd}$ :YAG laser with a pulse width of $8 \mathrm{~ns}, 300 \mathrm{~mW}$ average power and $300 \mu \mathrm{J}$ energy per pulse at $1064 \mathrm{~nm}$ wavelength and repetition rate of $1 \mathrm{kHz}$. Due to the temporal profile of the laser, the excited ultrasonic waves have a theoretical bandwidth from DC to around $150 \mathrm{MHz}$. The laser beam is focused onto a line source using a cylindrical lens of $160 \mathrm{~mm}$ focal distance, decreasing energy density by distributing laser power over a larger area compared to the focusing from a spherical lens.

The generation laser is set up in a Z-fold configuration as shown in Fig. 2. A stationary mirror (M1) and a galvo mirror system (GVS302, Thorlabs) controlled by a computer via a DAQ unit (USB-6001, National Instruments) are used to steer the beam onto the sample. The rotation of the galvo mirror changes the incidence angle of the generation laser's beam on the surface, allowing for the scanning of the generation laser. This system provides faster scanning compared to the scanning of the laser head by a motorized stage due to the lower inertia of the galvo mirror.

The out-of-plane ultrasonic component of the various excited waves is detected by a laser interferometer (Quartet, Sound \& Bright), capable of measuring on rough surfaces. The detection system utilized a second harmonic Nd:YAG continuous wave laser, at $532 \mathrm{~nm}$ wavelength with an average power on the sample of $780 \mathrm{~mW}$.

\section{B. Sample}

The component inspected was fabricated using the plasma arc Wire Arc Additive Manufacturing (WAAM) process with a single bead deposition strategy [16]. The material of the component was Ti-6Al-4V, a titanium alloy. For the purpose of ultrasonic imaging, several side-drilled holes were created. Cylindrical reflectors were chosen in order to achieve good contrast, improving defect detection capabilities of the system and the inspection side was polished to improve signal detection by the interferometric detector.

Table 1 shows defect size, their vertical position compared to the surface on the inspection side and their horizontal position relative to the center of the array aperture. The test sample can be seen on Fig. 3, highlighting defects D1-D3. The dimensions of the component are 26x10x110 mm.

TABLE I. DEFECT CHARACTERISTICS

\begin{tabular}{|l|c|c|c|}
\hline $\begin{array}{c}\text { Defect } \\
\text { name }\end{array}$ & $\begin{array}{c}\text { Defect location } \boldsymbol{X} \\
\text { (relative to array center) }\end{array}$ & Defect location $\boldsymbol{Y}$ & Diameter \\
\hline D1 & $8 \mathrm{~mm}$ & $5 \mathrm{~mm}$ & $1 \mathrm{~mm}$ \\
\hline D2 & $-2 \mathrm{~mm}$ & $7.5 \mathrm{~mm}$ & $1 \mathrm{~mm}$ \\
\hline D3 & $-12 \mathrm{~mm}$ & $10 \mathrm{~mm}$ & $1 \mathrm{~mm}$ \\
\hline
\end{tabular}

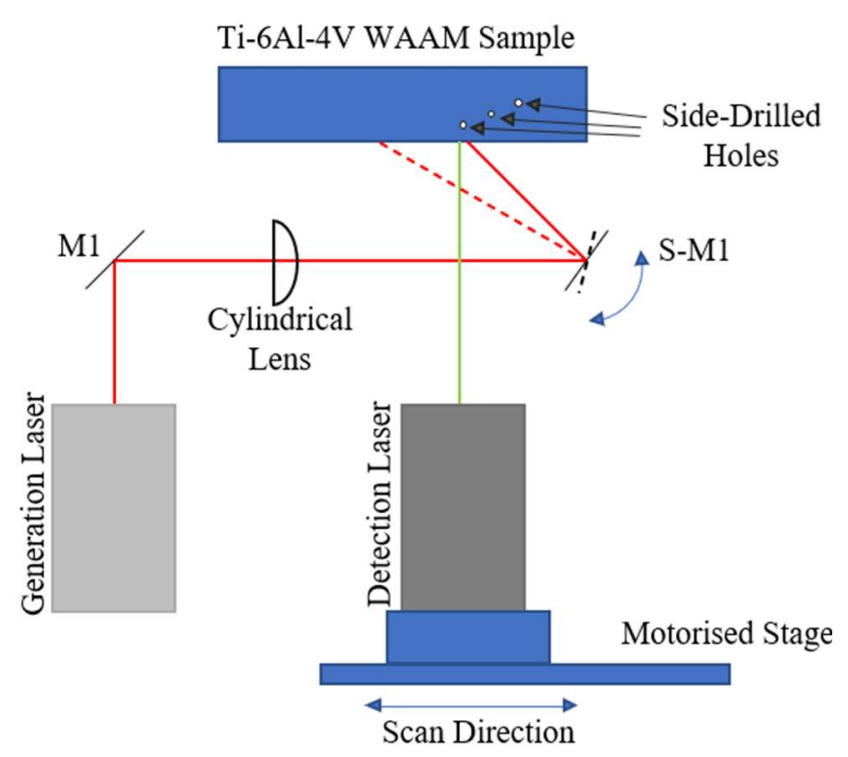

Fig. 2. Experimental setup showing generation and detection lasers, scanning stage, galvo mirror (S-M1) and the Titanium alloy WAAM sample.

\section{Synthesised Array and Digital Filtering}

The location of the array aperture is highlighted on Fig. 3. Aperture size is designed to be $16.75 \mathrm{~mm}$ and consisted of 68 elements with $250 \mu \mathrm{m}$ interelement spacing. Digital filtering is applied to the captured broadband A-scan signals to lessen the effect of frequency contents that reduce the SNR of the overall image.

In order to avoid the generation of grating lobes, array pitch must be smaller than half the wavelength [17]. This limits the system to wavelengths of $0.5 \mathrm{~mm}$ or larger. In this particular case, grating lobes do not appear below $6.6 \mathrm{MHz}$ for shear waves and $12.2 \mathrm{MHz}$ for longitudinal waves as the wavelength are shorter than the $0.5 \mathrm{~mm}$ limit for these frequencies.

\section{Results}

Fig. 4. shows the normalized TFM images that were processed and produced by (4) and (5), using shear and longitudinal modes. On these images, defects D1-D3 are clearly detectable with good contrast. In addition, the location of the defects matches their true location as described in Table 1 .

Laser generation of ultrasound excites both bulk and surface acoustic waves (SAWs). Crosstalk from SAWs into

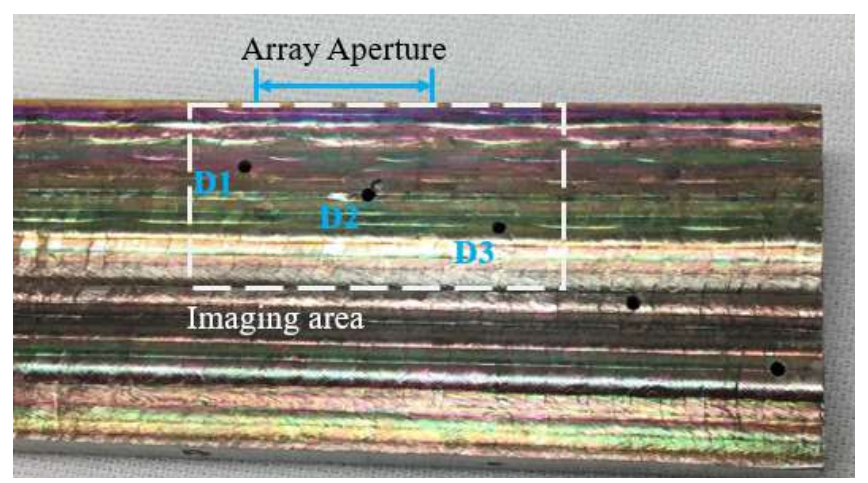

Fig. 3. Titanium WAAM sample, side-view. Generation and detection lasers were scanned on the top surface, indicated as 'Array Aperture'. White rectangule shows area imaged using bulk waves. 


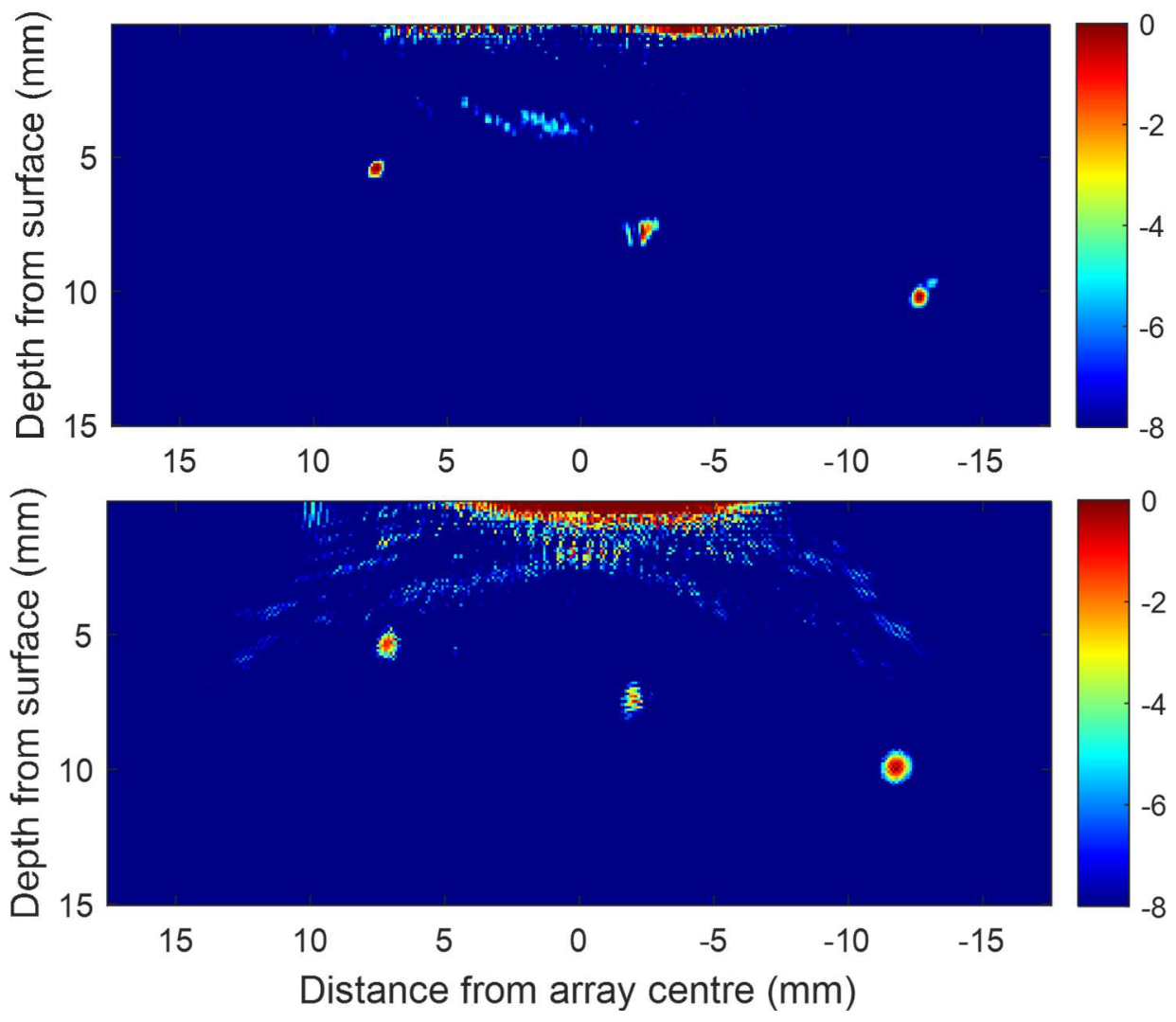

Fig. 4. TFM images of WAAM Ti-6Al-4V titanium alloy using ultrasonic shear (top image) and longitudinal (bottom image) waves. Digital Guassian filtering was used, at center frequencies 5.4 and $6.3 \mathrm{MHz}$, respectively for each TFM image. The colourbar on the right of each image shows the dynamic range used (dB scale).

shear and longitudinal TFM images can be observed as highintensity regions at the top of the images in Fig. 4, preventing useful interpretation of data in these areas. Both images in Fig. 4 are normalized to the corresponding largest defect response, rather than the very high amplitude SAW artefacts present, then are converted to $\mathrm{dB}$ scale for better visualization of the defects.

TFM, like other imaging techniques, is also not immune to the noise present in the acquired signals. To maximize the SNR of the captured data, signal averaging was performed. During data acquisition each captured waveform is averaged 128 times, requiring 14 minutes to capture the Full Matrix for the 68 element LIPA synthesized. The laser detector has a frequency limit of 1-66 MHz and during post-processing further digital filtering is applied in the form of a Gaussian filter of $100 \%$ bandwidth at $-40 \mathrm{~dB}$ and center frequency of 5.5 and $6.3 \mathrm{MHz}$ for shear and longitudinal waves respectively.

The sensitivity images, $E(x, z)$, were calculated for shear and longitudinal waves (Fig. 5). These images indicate how sensitive the system is to a defect at any given pixel of the image. The location of defects D1-D3 fall onto areas with good sensitivity for both shear and longitudinal cases and thus it is possible to image these defects accurately.

\section{DISCUSSION}

The images presented in the results section clearly demonstrate that high quality ultrasonic imaging can be achieved by using the TFM algorithm, both with shear and longitudinal modes. In the future, these modes, as well as images from mode converted waves could be used to perform data fusion [15], [18]. By performing this data fusion, the combined image has the potential for more accurate detection and characterization of the defects in the resulting image, minimising the effect of blind spots associated with individual wave modes [15] and further reducing the requirement for signal averaging during data acquisition. This could lead to faster LIPA data acquisition, which will be necessary for certain applications such as in-process inspection of WAAM.
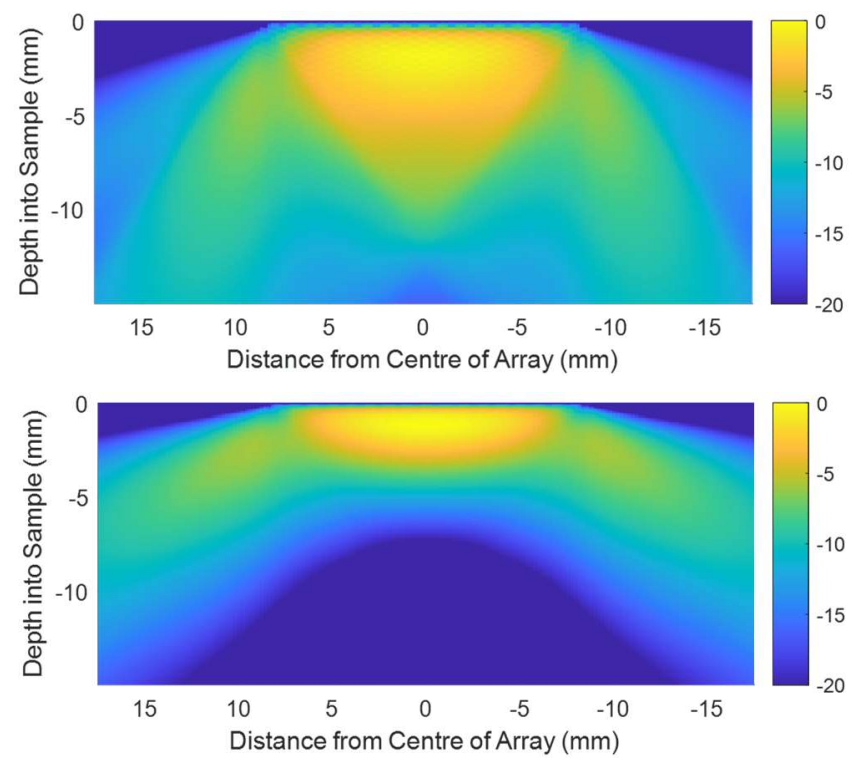

Fig. 5. Sensitivity images of 68 element LIPA, with $0.25 \mathrm{~mm}$ pitch on a titanium sample for shear waves (top image) and longitudinal waves (bottom image) demonstrating how sensitive the system is to a defect at any given pixel 
As mentioned in a previous section, the titanium alloy test object was polished in order to increase the proportion of reflected laser beam returning to the interferometric detectorhead in order to improve signal amplitude. While in this case LIPA imaging was not carried out on a rough surface, the detector used in this experimental setup is capable of inspecting on rough surfaces [19]. The rough surface is expected to reduce SNR, which would increase the averaging required during data acquisition, reducing the speed of the process. Ultimately, a trade-off will be determined between surface finish and inspection performance and time, in order to inspect as-deposited WAAM samples which would lead to in-process inspection during the component building process.

Although image qualities achieved by LIPAs are superior to that of previous laser ultrasonic imaging techniques, the ultrasonic array requires a considerable amount of time to capture the Full Matrix due to: a) the high number of A-scan signals captured, b) the signal averaging required to overcome the low amplitudes of laser ultrasonics and c) the mechanical scanning of the laser beams. It is noted here that 14 minutes were required to capture the Full Matrix for the dataset used in the TFM images shown in Fig. 4. To address this issue and enable industrial adoption of the technique for WAAM inprocess monitoring, it has been shown theoretically that it is possible to perform an optimized, adaptive scan based on the sensitivity images shown in Fig. 5 [20]. The referenced technique was able to produce images with same SNR as the ones produced by FMC but captured 10 times less data. Applying the same scanning method for the case presented in this paper would have reduced the data acquisition time from 14 minutes to 1 minute 20 seconds.

It was previously pointed out that noise is present on TFM images. This noise can be categorised either as incoherent or coherent noise. The former can arise from various sources, some of which can include environmental vibration, optical and electrical interference and thermal noise, while coherent noise mainly occurs due to internal features of the sample that are not of interest, such as the microstructure. As incoherent noise generally appears at random parts of the signal, the TFM algorithm significantly reduces their effect [21]. Furthermore, by applying signal averaging, incoherent noise can be further reduced. While in this paper no additional effort was made to overcome the coherent noise caused by the scattering of the sample, it is demonstrated that LIPAs can produce ultrasonic images even in a highly scattering titanium alloy. In future work, image quality could be improved by quantifying the scattering noise, as described in [22], to compensate for it.

\section{CONCLUSION}

In this paper, remote, laser ultrasonic imaging of a WAAM sample is presented using a Laser Induced Phased Array. The titanium alloy sample was scanned by a single pulsed generation laser and a laser interferometric detection system to synthesise the LIPA. TFM images were produced of a titanium alloy sample, with defects up to $10 \mathrm{~mm}$ depth clearly detected. This demonstrates that LIPAs can produce highquality images of internal features even in materials of highly scattering medium. Thus, LIPAs can provide a way for inprocess inspection for WAAM if the data acquisition stage is reduced to below a second. This is the target of our studies currently and will provide the possibility of reducing defective AM components and correcting defects during AM.

\section{ACKNOWLEDGMENT}

The authors would like to express their gratitude to $\mathrm{R}$. Bettie and $\mathrm{H}$. Anderson for their continual support in keeping the lab a productive environment. Furthermore, the authors would like to thank Connor Ronald Doherty for his assistance and support in producing this paper.

\section{REFERENCES}

[1] M. Cotteleer, and J. Joyce, "3D opportunity: Additive manufacturing paths to performance, innovation, and growth," Deloitte Review, vol. 14, 2014.

[2] Y. Javadi, C. N. MacLeod, S. G. Pierce, A. Gachagan, D. Lines, C. Mineo, J. Ding, S. Williams, M. Vasilev, E. Mohseni, and R. Su, "Ultrasonic phased array inspection of a Wire + Arc Additive Manufactured (WAAM) sample with intentionally embedded defects", Addit. Manuf., vol. 29, 1008060, 2019.

[3] Y. Javadi, C. N. MacLeod, S. G. Pierce, A. Gachagan, W. Kerr, J. Ding, S. Williams, M. Vasilev, R. Su, C. Mineo, and J. Dziewierz, "Ultrasonic phased array inspection of wire plus arc additive manufacture (WAAM) samples using conventional and total focusing method (TFM) imaging approaches”, Insight, vol. 61, no. 3, pp. 144$148,2019$.

[4] C. B. Scruby, and L. E. Drain, "Laser Ultrasonics Techniques and Applications", Bristol, UK, CRC Press, 1990

[5] C. B. Scruby, "Some applications of laser ultrasound", Ultrasonics, vol. 27, no. 4, 1989.

[6] D. Lévesque, C. Bescond, M. Lord, X. Cao, P. Wanjara, and J.-P. Monchalin, "Inspection of additive manufactured parts using laser ultrasonics", AIP Conference Proceedings, vol. 1706, 130003, 2016.

[7] D. Cerniglia, M. Scafidi, A. Pantano, and J. Rudlin, "Inspection of additive-manufactured layered components" Ultrasonics, vol. 62, pp. 292-298, 2015.

[8] G. Davis, R. Nagarajah, S. Palanisamy, R. A. R. Rashid, P. Rajagopal, and K. Balasubramaniam, "Laser ultrasonic inspection of additive manufactured components", Int. J. Adv. Manuf. Tech., vol. 102, pp. 2571-2579, 2019

[9] D. Pieris, T. Stratoudaki, Y. Javadib, P. Lukacs, S. Catchpole-Smith, P. D. Wilcox, A. Clare, and M. Clark, "Laser Induced Phased Arrays (LIPA) to detect nested features in additively manufactured components", Mater. Des., vol. 187, 108412, 2020.

[10] T. Stratoudaki, M. Clark, and P. D. Wilcox, "Laser induced ultrasonic phased array using full matrix capture data acquisition and total focusing method", Opt. Express, vol. 24, no. 19, pp. 21921-21938, 2016.

[11] S. J. Davies, C. Edward, G. S. Taylor, and S. B. Palmer, "Lasergenerated ultrasound: its properties, mechanisms and multifarious applications", J. Phys. D: Appl. Phys., vol. 26, no. 329, 1993.

[12] L. R. F. Rose, "Point-source representation for laser-generated ultrasound", J. Acoust. Soc. Am., vol. 75, no. 723, 1984.

[13] C. Holmes, B. W. Drinkwater, and P. D.Wilcox, "Post-processing of the full matrix of ultrasonic transmit-receive array data for nondestructive evaluation", NDT\&E Int., vol. 38, no. 8, pp. 701-711, 2005.

[14] C. Fan, M. Caleap, M. Pan, and B. W. Drinkwater, "A comparison between ultrasonic array beamforming and super resolution imaging algorithms for non-destructive evaluation", Ultrasonics, vol. 54, pp. 1842-1850, 2014.

[15] T. Stratoudaki, M. Clark, and P. D. Wilcox, "Adapting the full matrix capture and the total focusing method to laser ultrasonics for remote non destructive testing", IEEE Int. Ultrason. Symp., pp. 3-6, 2017.

[16] S. W. Williams, F. Martina, A. C. Addison, J. Ding, G. Pardal, and P. Colegrove, "Wire + Arc Additive Manufacturing", Mater. Sci. Technol., vol. 32, no. 7, pp. 641-647, 2016.

[17] S.-C. Wooh, and Y. Shi, "Optimum beam steering of linear phased arrays", Wave Motion, vol. 29, no. 3, pp. 245-265, 1999.

[18] P. D. Wilcox, A. J. Croxford, N. Budyn, R. L. T. Bevan, J. Zhang, A. Kashubin, and P. Cawley, "Fusion of multi-view ultrasonic data for increased detection performance in non-destructive evaluation", Proc. R. Soc. A., vol. 476, 20200086, 2020.

[19] T. Blum, B. Pouet, S. Breugnot, and P. Clémenceau, "Non-destructive testing using multi-channel random-quadrature interferometer", AIP Conf. Proc., vol. 975, no. 239, 2008. 
[20] P. Lukacs, T. Stratoudaki, P. Wilcox, M. Clark, and A. Gachagan, "Optimisation of data acquisition and processing for laser induced ultrasonic phased arrays”, Proc. Mtgs. Acoust. vol. 38, 0300152019.

[21] P. D. Wilcox, "Ultrasonic arrays in NDE: Beyond the B-scan", AIP Conf. Proc., vol. 1511, no. 33, 2013.
[22] R. L. T. Bevan, J. Zhang, N. Budyn, A. J. Croxford, and P. D Wilcox, "Experimental Quantification of Noise in Linear Ultrasonic Imaging", IEEE Trans. Ultrason., Ferroelectr., Freq. Control, vol. 66, no. 1, pp. 79-90, 2019. 
2021-06-28

\title{
Remote ultrasonic imaging of a wire arc additive manufactured Ti-6AI-4V
}

component using laser induced phased array

\author{
Lukacs, Peter
}

IEEE

Lukacs P, Davis G, Stratoudaki T, et al., (2021) Remote ultrasonic imaging of a wire arc additive manufactured Ti-6AI-4V component using laser induced phased array. In: 2021 IEEE International Instrumentation and Measurement Technology Conference (I2MTC), 17-20 May 2021, Glasgow

https://doi.org/10.1109//2MTC50364.2021.9459823

Downloaded from Cranfield Library Services E-Repository 